\title{
Static analysis and parameters synthesis of planetary-layshaft transmissions with three power flows
}

\author{
Konstantin Salamandra ${ }^{1, *}$ \\ ${ }^{1}$ Mechanical Engineering Research Institute of the Russian Academy of Sciences (IMASH RAN), \\ 101990, M. Kharitonyevsky per. 4, Moscow Russia
}

\begin{abstract}
The increase in the number of transmission ratios in the vehicles gearboxes leads to an increase in the number of mechanisms used and their complication. One way to obtain simple designs of multi-speed transmissions with smaller number of gears, clutches, brakes and synchronizers is to use combinations of planetary differential gears and gears with fixed axes (planetary-layshaft transmissions). The article presents a static analysis of a planetary-layshaft transmissions with three parallel power flows for obtaining the dependencies between the gear ratios of the internal mechanisms and the speed ratios of the transmission. A technique for determination the gear ratios of the internal mechanisms for providing a close to a given series of transmission ratios is described.
\end{abstract}

\section{Introduction}

The best way to reduce the fuel consumption and improve the automotive efficiency is to reduce the speed range of the internal combustion engine used [1-4]. The more transmission ratios implemented by gearbox the more complicated the transmission is. The three-stream transmission designs were presented in [5], the designs consist of two differentials and gears with fixed axles. Obtained designs permit larger number of speeds by controlling the operating modes of differentials, while they consist of simple mechanisms and have high efficiency. Compared to the currently used 8 to 10 -speed planetary transmissions and 7 -speed dual-clutch transmissions the planetary-layshaft transmissions have fewer gears and shifting elements (clutches, brakes and synchronizers) herewith realize a larger number of speeds. In addition, due to the splitting the power flow between the three branches a significant reduction in the loads on the transmission members and control elements is achieved. The possible structures of transmissions with three power-flows consisted of generalized diagrams [6-8] were presented in [5]. Obtained structures were classified in dependence of the location the differentials, input and output members (DD, DDO, IDD, and IDDO).

The paper is targeted to describing the technic for determination the gear ratios of internal mechanisms for providing a set of transmission ratios of the gearboxes with three power flows close to the specified.

\footnotetext{
*Corresponding author: ksalamandra@yandex.ru
} 
As an example, let's consider the IDDO structure of the planetary-layshaft transmission in which both differentials are located in the inner contours, and the movement is distributing on the input member I and summing up at the output member O (Fig. 1).

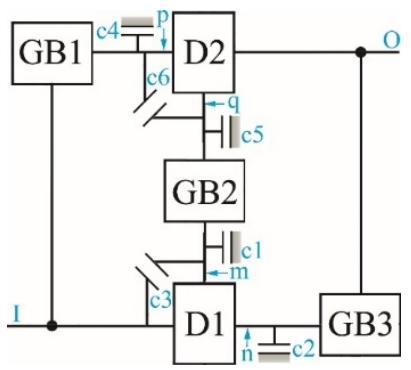

Fig. 1. The IDDO structure of the planetary-layshaft transmission with three power flows.

I, O - input and output members consequently; GB1, GB2, GB3 - layshaft gear sets with built-in shifting elements which switch-on the internal gears; D1, D2 - differential gears; $m, n$ - members of differential D1; p, q- members of differential D2; c1 - c6 - shifting elements of differentials D1 and D2.

Depending on the condition of the $\mathrm{c} 1-\mathrm{c} 6$ the transmission operates in three modes: onestream, two-stream and three-stream. The transmission has four degrees of freedom, i.e. 3 shifting elements must be switched on at each speed. At the same time in one-stream modes of GB1 or GB3 only two shifting elements are activated - one in the corresponding GB and one element in one of the differentials $\mathrm{D}$. This property is a characteristic feature of the selected structure, extends the functionality of the transmission and can be used for preparation the next speed, i.e. preselect the next shifting elements as in the dual-clutch transmissions [9]. In addition, at these speeds the third shifting element may be switched on for minimizing losses at idle rotation.

\section{Static analysis}

The transmission ratio from I to $\mathrm{O}\left(i_{I O}\right)$ on one-stream modes depending on the switched-on clutches for each GBs are shown in Table 1.

Table 1. One-stream modes transmission ratios of IDDO structure.

\begin{tabular}{|c|c|c|c|}
\hline \multicolumn{2}{|c|}{ Switched-on transmission elements } & $\boldsymbol{i}_{\boldsymbol{I O}}$ \\
\hline $\mathrm{GB} 1$ & - & $\mathrm{c} 5$ & $i_{G B 1} / i_{O p}^{q}$ \\
\hline $\mathrm{GB} 2$ & $\mathrm{c} 2$ & $\mathrm{c} 4$ & $i_{G B 2} i_{I m}^{n} / i_{O q}^{p}$ \\
\hline $\mathrm{GB} 2$ & $\mathrm{c} 2$ & $\mathrm{c} 6$ & $i_{G B 2} i_{I m}^{n}$ \\
\hline $\mathrm{GB} 2$ & $\mathrm{c} 3$ & $\mathrm{c} 4$ & $i_{G B 2} / i_{O q}^{p}$ \\
\hline $\mathrm{GB} 3$ & $\mathrm{c} 1$ & - & $i_{G B 3} i_{I n}^{m}$ \\
\hline $\mathrm{GB} 1$ & - & $\mathrm{c} 6$ & $i_{G B 1}$ \\
\hline $\mathrm{GB} 2$ & $\mathrm{c} 3$ & $\mathrm{c} 6$ & $i_{G B 2}$ \\
\hline $\mathrm{GB} 3$ & $\mathrm{c} 3$ & - & $i_{G B 3}$ \\
\hline
\end{tabular}

Two-stream modes in the IDDO structure via GB1 and GB2 when switched-on c2 or c3, and two-stream modes via GB2 and GB3 when switched-on c4 or c6 are possible. Twostream mode via GB1 and GB3 is not possible in this structure. To calculate the transmission ratio of two-stream modes, let's perform a static analysis of the IDDO structure at switchedon clutch c3 (Fig. 2a) and c6 (Fig. 2b). The elements of the structure that do not operate in two-stream modes are not shown in Figure 2. 


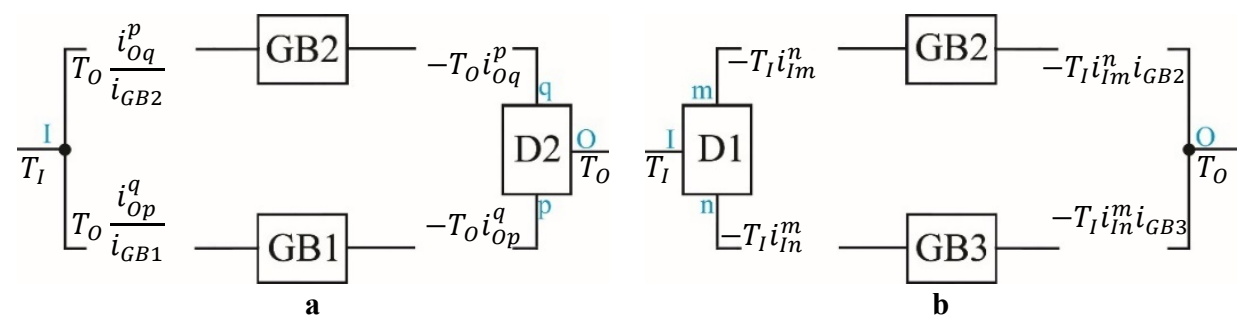

Fig. 2. Static analysis of IDDO structure in two-stream operation modes: a - clutch c3 is switched-on; $\mathrm{b}$ - clutch $\mathrm{c} 6$ is switched-on.

The letter $i$ with the corresponding subscript indicates the gear ratio of each GBs. The left lower subscript in the gear ratios of the differentials is the member assumed as driver; the right lower subscript is the member assumed as driven; the superscript is the member assumed as fixed.

Based on the static equilibrium of the input member I (Fig. 2a), we obtain:

$$
i_{I O}=-T_{O} / T_{I}=1 /\left(i_{O p}^{q} / i_{G B 1}+i_{O q}^{p} / i_{G B 2}\right)
$$

From the static equilibrium of the output member O (Fig. 2b):

$$
i_{I O}=i_{I m}^{n} i_{G B 2}+i_{I n}^{m} i_{G B 3}
$$

Similarly, the transmission ratio $i_{I O}$ via GB1 and GB2 when brake $\mathrm{c} 2$ is switched-on:

$$
i_{I O}=1 /\left(i_{O p}^{q} / i_{G B 1}+i_{O q}^{p} /\left(i_{G B 2} i_{I m}^{n}\right)\right)
$$

Transmission ratio via GB2 and GB3 when brake c4 is switched-on:

$$
i_{I O}=i_{G B 2} i_{I m}^{n} / i_{O q}^{p}+i_{G B 3} i_{I n}^{m}
$$

The static analysis of the three-stream mode of the IDDO structure is shown in Fig. 3. In this mode all differential clutches are switched-off.

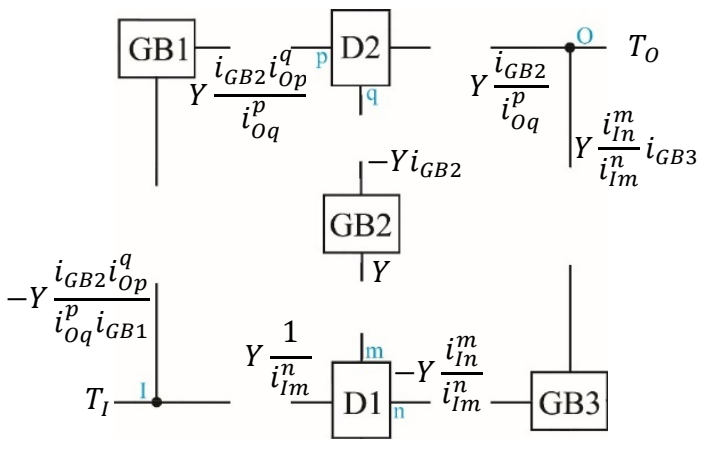

Fig. 3. Static analysis of IDDO structure in three-stream operation mode.

A static equilibrium at the input $\mathrm{I}$ and output $\mathrm{O}$ results in a system of two equations from which we obtain:

$$
i_{I O}=-\frac{T_{O}}{T_{I}}=i_{G B 1} \frac{i_{G B 2} i_{I m}^{n}+i_{I n}^{m} i_{O q}^{p} i_{G B 3}}{i_{G B 2} i_{O p}^{q} i_{I m}^{n}+i_{O q}^{p} i_{G B 1}}
$$


In the IDDO structure (Fig. 1) the maximum number of differential control elements (brakes c1, c2, c4, c5 and locking clutches c3, c6), which determine the possible two and one-stream modes, are presented. In specific kinematic diagrams of gearboxes these control elements may be absent, then this will reduce the number of modes of such transmission.

It is possible to construct a set of kinematic diagrams of multi-speed transmissions according to structure in Fig. 1, in Fig. 4 one of the possible kinematic diagram is presented. There are GB1, GB2, GB3 with two gears and synchronizers s7, s8, s9 which switching on appropriate GBs. There are present only clutches $\mathrm{c} 3$ and c6 among the control elements of differentials. Clutches shift lead the differential locking and it rotation with direct gear ratio. The gearbox is not coaxial, the rotation of the input $\mathrm{I}$ and output $\mathrm{O}$ shafts are in opposite direction. In order to increase the gear ratio from the mechanism to the mechanism in the gearbox the input of differential D1 is connected to the sun pinion, because the gear ratio from the sun to the ring gear and carrier is greater than 1 . The rotation direction of the carrier will coincide with the rotation direction of the sun in this case, and the ring gear will rotate in the opposite direction. If the planetary gear set will be blocked with the clutch c3, all parts of the D1 will rotate in the same direction as the sun. Therefore, GB1 connected to the ring gear D1 has two gears. The first - with a parasitic gear for unidirectional rotation of all parts of the gearbox on three- and two-stream modes when the clutch c6 is switched-on, as well as to obtain reverse gear when the $\mathrm{c} 3$ and $\mathrm{c} 6$ are switched-on. The second gear of GB1 consists of two wheels and operates when the clutch c3 is switched-on. Thus transmission (Fig. 4) can realize 6 one-stream, 4 two-stream and 4 three-stream speeds. The total speeds in transmission (Fig. 4) are 14, one of which is reverse gear. Reverse gear is carried out when the clutches $\mathrm{c} 3, \mathrm{c} 6$ are switched-on and synchronizer $\mathrm{s} 8$ are switched to position $\mathrm{b}$.

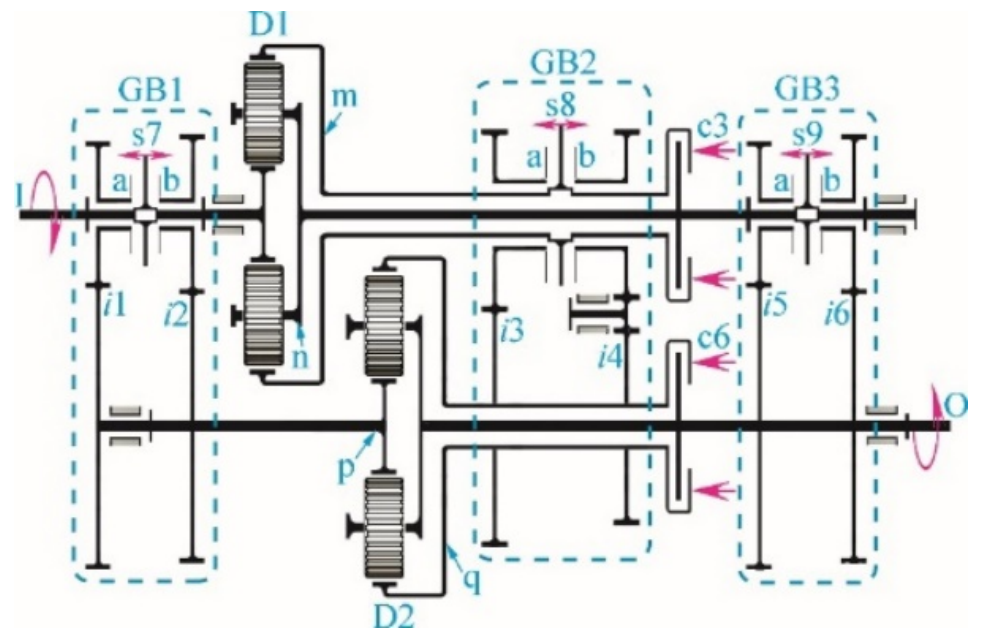

Fig. 4. Kinematic diagram of IDDO transmission.

\section{Parameters synthesis}

Using the transmission design in Fig. 4 as an example, we demonstrate the solution of the problem of parametric synthesis, which consists in determining the internal gear ratios of the planetary gears and the sets of gears GB1, GB2 and GB3 providing a given or close to the given $a_{k}$ series of $i_{I O}$ gear ratios:

$$
a_{k}=a_{1}, a_{2}, \ldots, a_{13}
$$

where $k$ is the speed number. 
Moreover, the terms of the $a_{k}$ series have negative values, because the direction of rotation of the output shaft $\mathrm{O}$ is opposite to the direction of rotation of the shaft $\mathrm{I}$ and, accordingly, $a_{k}<a_{k+1}$.

There are 8 independent parameters in the gearbox (Figure 4): $i 1, i 2, i 3, i 4, i 5, i 6, i_{I m}^{n}$, $i_{o p}^{q}$. Moreover, in accordance with the properties of the differential $[6,7]: i_{I m}^{n}=1-i_{I n}^{m}$; $i_{O p}^{q}=1-i_{O q}^{p}$.

Thus, the problem of parametric synthesis of a transmission consists in determining the values of the parameters $i 1, i 2, i 3, i 4, i 5, i 6, i_{I m}^{n}, i_{o p}^{q}$, at which a number of transmission ratios $a_{k}$ satisfying formulas (6-13) are achieved. If the terms of the given series $a_{k}$ (14) do not satisfy formulas $(6-13)$, then there is no transmission ideally satisfying the series (14). But one can try to find some series close to (14) by searching for the values $i 1, i 2, i 3, i 4, i 5$, $i 6, i_{I m}^{n}, i_{O p}^{q}$, at which the smallest deviations of the terms of the obtained series $b_{k}$ from the set values $a_{k}$. Therefore, the synthesis problem for the transmission under investigation is divided into the following consecutive stages:

1. Due to the change in the parameters $i 1, i 2, i 3, i 4, i 5, i 6, i_{I m}^{n}, i_{o p}^{q}$, the series of transmission ratios $i_{I O}$ closest to (14) is revealed. The closeness of the series will be estimated from the minimum of the function:

$$
F_{1}=\sum_{k=1}^{13}\left(\frac{a_{k}-b_{k}}{a_{k}}\right)^{2}
$$

where $F_{1}$ is a function that depends only on the gear ratios $i 1, i 2, i 3, i 4, i 5, i 6, i_{I m}^{n}, i_{O p}^{q}$; $b_{k}$ are terms of the series that satisfy expressions (6 - 13).

2. The relative deviations $\alpha$ of the values of the transmission ratios of the obtained series $b_{k}$ and the relative deviations of the step $\beta$ are calculated:

$$
\alpha=\frac{a_{k}-b_{k}}{a_{k}} ; \beta=1-\frac{a_{k+1} \cdot b_{k}}{a_{k} \cdot b_{k+1}}
$$

If the relative deviations of the transmission ratios are satisfactory, then the problem of parametric synthesis can be considered as solved. If the average values are $\beta>\alpha$, then to obtain a more uniform series of transmission gear ratios, it is necessary to consider the minimum of the sum of the squares of the relative deviations of the series $b_{k} / b_{k+1}$ from the series $a_{k} / a_{k+1}$, i.e. the deviation of the step between the ratios of the received series from the step between the ratios of the given series:

$$
F_{2}=\sum_{k=1}^{13}\left(\frac{\frac{a_{k}}{a_{k+1}}-\frac{b_{k}}{b_{k+1}}}{\frac{a_{k}}{a_{k+1}}}\right)^{2}=\sum_{k=1}^{13}\left(1-\frac{a_{k+1} \cdot b_{k}}{a_{k} \cdot b_{k+1}}\right)^{2}
$$

The obtained values of the gear ratios of internal mechanisms are the solution of the task.

In order to obtain a rational transmission size, we restrict ourselves to the recommended range [10] for changing gear ratios for gears with fixed axles from -3 to -0.4 and planetary gears from -4 to -1.5 .

Let's illustrate the described technique with an example, let a series $a_{k}$ be given (the first column in Table 2). As a result of minimizing the criterion (15), we obtained the series $b_{k}^{1}$ (the second column in Table 2). 
Table 2. The given and obtained series of transmission ratios after minimization by criterion (15).

\begin{tabular}{|c|c|c|c|c|c|c|c|}
\hline \multicolumn{3}{|c|}{ Given series $\boldsymbol{a}_{\boldsymbol{k}}$} & \multicolumn{3}{c|}{ Obtained series $\boldsymbol{b}_{\boldsymbol{k}}^{\mathbf{1}}$} & $\boldsymbol{\alpha}, \boldsymbol{\%}$ & $\boldsymbol{\beta}, \boldsymbol{\%}$ \\
\hline \multicolumn{2}{|c|}{$\boldsymbol{i}_{\boldsymbol{I O}}$} & Step & \multicolumn{2}{|c|}{$\boldsymbol{i}_{\boldsymbol{I} \boldsymbol{O}}$} & Step & & \\
\hline$a_{1}$ & $-9,16$ & & $b_{1}$ & $-8,123$ & & 11,3 & \\
\hline$a_{2}$ & $-7,00$ & 1,3 & $b_{2}$ & $-7,156$ & 1,135 & 2,2 & 13,3 \\
\hline$a_{3}$ & $-5,42$ & 1,3 & $b_{3}$ & $-5,299$ & 1,350 & 2,2 & 4,6 \\
\hline$a_{4}$ & $-4,17$ & 1,3 & $b_{4}$ & $-4,865$ & 1,089 & 16,7 & 16,2 \\
\hline$a_{5}$ & $-3,20$ & 1,3 & $b_{5}$ & $-3,00$ & 1,622 & 6,3 & 24,4 \\
\hline$a_{6}$ & $-2,50$ & 1,3 & $b_{6}$ & $-2,25$ & 1,333 & 10,0 & 4,2 \\
\hline$a_{7}$ & $-1,9$ & 1,3 & $b_{7}$ & $-1,928$ & 1,167 & 1,5 & 11,3 \\
\hline$a_{8}$ & $-1,46$ & 1,3 & $b_{8}$ & $-1,285$ & 1,500 & 12,0 & 15,3 \\
\hline$a_{9}$ & $-1,12$ & 1,3 & $b_{9}$ & $-1,18$ & 1,089 & 5,4 & 16,5 \\
\hline$a_{10}$ & $-0,864$ & 1,3 & $b_{10}$ & $-0,913$ & 1,292 & 5,7 & 0,3 \\
\hline$a_{11}$ & $-0,72$ & 1,2 & $b_{11}$ & $-0,833$ & 1,096 & 15,7 & 8,7 \\
\hline$a_{12}$ & $-0,60$ & 1,2 & $b_{12}$ & $-0,573$ & 1,454 & 4,5 & 21,1 \\
\hline$a_{13}$ & $-0,50$ & 1,2 & $b_{13}$ & $-0,45$ & 1,273 & 10,0 & 6,1 \\
\hline
\end{tabular}

The values of gear ratios of gearbox mechanisms (Figure 4), at which the series $b_{k}^{1}$ is obtained: $i 1=-0,45 ; i 2=-3 ; i 3=-1,928 ; i 4=3 ; i 5=-0,913 ; i 6=-0,573 ; i_{I m}^{n}=$ $-1,842 ; i_{o p}^{q}=0,4$. The average value of $\alpha_{a m}=8,6 \%$, and the average value of $\beta_{a m}=$ $12,9 \%$. Because $\beta_{a m}>\alpha_{a m}$, we carry out the minimization by criterion (16). The obtained result is in Table 3.

Table 3. The obtained series of transmission ratios after minimization by criterion (16).

\begin{tabular}{|c|c|c|c|c|c|c|c|c|c|c|c|}
\hline \multirow{3}{*}{ Speed } & \multicolumn{8}{|c|}{ Control elements condition } & \multirow{3}{*}{$i_{I O}$} & \multirow{3}{*}{ Step } & \multirow{3}{*}{$\beta, \%$} \\
\hline & \multicolumn{2}{|c|}{ s7 } & \multicolumn{2}{|c|}{ s8 } & \multicolumn{2}{|c|}{ s9 } & \multirow{2}{*}{ c3 } & \multirow{2}{*}{ c6 } & & & \\
\hline & $\mathbf{a}$ & $\mathbf{b}$ & $\mathbf{a}$ & $\mathbf{b}$ & $\mathbf{a}$ & $\mathbf{b}$ & & & & & \\
\hline 1. & & & & $\mathrm{x}$ & $\mathrm{x}$ & & & $\mathrm{x}$ & $-6,707$ & & \\
\hline 2. & & & & $\mathrm{x}$ & & $\mathrm{x}$ & & $\mathrm{x}$ & $-5,52$ & 1,215 & 7,1 \\
\hline 3. & & $\mathrm{x}$ & & $\mathrm{x}$ & $\mathrm{x}$ & & & & $-4,546$ & 1,214 & 6,0 \\
\hline 4. & & $\mathrm{x}$ & & $\mathrm{x}$ & & $\mathrm{x}$ & & & $-4,036$ & 1,126 & 13,3 \\
\hline 5. & & $\mathrm{x}$ & & & & & & $\mathrm{x}$ & $-2,917$ & 1,384 & 6,2 \\
\hline 6. & & $\mathrm{x}$ & $\mathrm{x}$ & & & & $\mathrm{x}$ & & $-2,457$ & 1,187 & 7,2 \\
\hline 7. & & & $\mathrm{x}$ & & & & $\mathrm{x}$ & $\mathrm{x}$ & $-2,063$ & 1,191 & 9,5 \\
\hline 8. & $\mathrm{x}$ & & & $\mathrm{x}$ & $\mathrm{x}$ & & & & $-1,729$ & 1,193 & 8,3 \\
\hline 9. & $\mathrm{x}$ & & & $\mathrm{x}$ & & $\mathrm{x}$ & & & $-1,535$ & 1,126 & 13,6 \\
\hline 10. & & & & & $\mathrm{X}$ & & $\mathrm{x}$ & & $-1,316$ & 1,166 & 10,0 \\
\hline 11. & $\mathrm{x}$ & & $\mathrm{x}$ & & & & $\mathrm{x}$ & & $-1,059$ & 1,243 & 3,6 \\
\hline 12. & & & & & & $\mathrm{x}$ & $\mathrm{x}$ & & $-0,872$ & 1,214 & 1,2 \\
\hline 13. & $\mathrm{x}$ & & & & & & & $\mathrm{x}$ & $-0,756$ & 1,153 & 3,9 \\
\hline 14. $\mathrm{R}$ & & & & $\mathrm{x}$ & & & $\mathrm{x}$ & $\mathrm{x}$ & 1,904 & & \\
\hline
\end{tabular}

The average value of the relative deviation of the step of the obtained series is $8.2 \%$. The gear ratios of the gearbox mechanisms: $i 1=-0,756 ; i 2=-2,917 ; i 3=-2,063 ; i 4=$ 1,$904 ; i 5=-1,316 ; i 6=-0,872 ; i_{I m}^{n}=-1,674 ; i_{O p}^{q}=0,549$.

The research was carried out under financial support from Russian Science Foundation, project No 1779-10493. 


\section{References}

1. G. Wagner, H. Naunheimer, H. Scherer et al., ATZ Automobiltech Z 109, 512 (2007)

2. H. Ota, K. Nozaki, A. Honda, M. Kinoshita et al., SAE Tech. P. 2007-01-1101 (2007).

3. J. Broge, ZF's nine-speed geared for front-transverse engines, URL: http://articles.sae.org/11884/

4. P. Dong, Y. Liu, P. Tenberge, X. Xu, Mech. and Mach. Theory, 108, 83 (2017)

5. K. Salamandra, Transmissions with three power-flows for trucks and tractors, Engineering for Rural Development Proceedings, 2018 (to be published)

6. D. Volkov, A. Kraynev, Transmissii stroitelnikh i dorojnikh mashin (Transmissions for construction and road machinery). Moscow, Mashinostroenie, (1974) (in Russian)

7. H. Müller, Die Umlaufgetriebe, Springer, Berlin, Heidelberg (1998)

8. A. Kraynev, K. Salamandra, M. Raghavan, Synthesis of the Two-Stream Transmissions. Power Transmissions. Mechanisms and Machine Science, Springer, Dordrecht (2013)

9. B. Matthes, SAE Tech. P. 2005-01-1021 (2005)

10. V. Kudryavtsev, Yu. Kirdyashev, Planetarniye peredachi (Spravochnik) (Planetary gears (Handbook)). Leningrad, Mashinostroenie, (1977) (in Russian) 